\title{
611.67 \\ puntos de vista sobre el contenido de yeso de los cementos portland
}

J. CALLEJA

Dr. en Ciencias Químicas

\section{resummem}

Se justifican, mediante los datos y observaciones expuestos en este trabajo, las modificaciones introducidas en el Pliego (Norma española) para conglomerantes hidráulicos de 1961 - PCCiH 61 - en relación con el antiguo Pliego de 1930 y sus revisiones hasta 1957, respecto del contenido de yeso de los cementos Portland, expresado por el valor máximo de $\mathrm{SO}_{3}$ admitido en los mismos.

Se justifica igualmente el mantenimiento de dichas modificaciones en la nueva y reciente versión del Pliego de 1964 - PCCH 64 - en la misma o muy parecida forma en que fueron originalmente introducidas, conservándose integramente el criterio que presidió su introducción.

Se demuestra que este criterio ha sido y sigue siendo adoptado por otras Normas de diversos países, después de su implantación con carácter original y de prioridad en el nuestro.

Se estima que, por existir indiscutibles y bien fundadas razones cientifico-técnicas, apoyadas por resultados experimentales, para adoptar y mantener tal criterio, no debe intentarse lo contrario, a menos que se aduzcan otras razones y otros hechos suficientemente convincentes para ello.

La consideración de que por ser, en general, el valor máximo de $\mathrm{SO}_{3}$, admitido en el Pliego español, superior a los correspondientes de otras Normas extranjeras, ello pudiera dificultar una posible concurrencia de los cementos españoles a los mercados internacionales, es inconsistente, por cuanto que dicho valor limitativo es, precisamente, un máximo maximorum. No debe olvidarse tampoco, a este respecto, que, por razón de las materias primas, muy pocos cementos Portland extranjeros, si es que hay alguno, poseen - en promedio- un contenido tan elevado de aluminato tricálcico como el de los cementos españoles.

\section{1. - antecedentes}

Hay temas y cuestiones, dentro de la doctrina y de la tecnología de los cementos, que, pese a considerarse abundantemente tratados en todo tiempo y, en consecuencia, creerse resueltos, son, cuando menos, poco conocidos y, a pesar de todo, muy propicios para emitir libre opinión sobre ellos. Uno de estos temas es el relativo al contenido de $\mathrm{SO}_{3}$ - imáxi- 
mo?, ¿máximo y mínimo?, ¿óptimo?- de los cementos en general, y de los de tipo Portland en particular.

Sobre el asunto ha tenido ocasión de investigar y experimentar el autor de este artículo desde 1948, y de publicar algunos trabajos originales desde 1956. El primero de ellos fue presentado en dicho año a la III Reunión Internacional sobre Reactividad de los Sólidos (1). En él se hacía una amplia introducción bibliográfica - 76 citas-, puesta al día a la sazón - a la que se remite al lector interesado - que se centraba sobre las relaciones entre el contenido de yeso $\left(\mathrm{SO}_{3}\right)$ de los cementos y su fraguado, resistencias y expansión, pero principalmente sobre su contenido de $\mathrm{C}_{3} \mathrm{~A}$ potencial y su retracción. Se glosaba el concepto de "cemento correctamente retardado" introducido por Lerch, así como el de "yeso óptimo" de un cemento, y se barajaban los valores "máximos" de $\mathrm{SO}_{3}$ impuestos por las Normas de diversos países. En el aspecto doctrinal, y como consecuencia de los resultados experimentales del trabajo, se expuso la tesis de que el contenido de yeso "óptimo" de un cemento Portland depende - a constancia de otros factores - fundamentalmente del contenido de $\mathrm{C}_{3 \mathrm{~s}} \mathrm{~A}$ del mismo, y precisamente en cuanto que dicho yeso puede formar con el aluminato tricálcico, el sulfoaluminato cálcico monosulfato hidratado, según la reacción:

$$
\mathrm{CaSO}_{4} \cdot 2 \mathrm{H}_{2} \mathrm{O}+3 \mathrm{CaO} \cdot \mathrm{Al}_{2} \mathrm{O}_{3}+10 \mathrm{H}_{2} \mathrm{O} \rightarrow 3 \mathrm{CaO} \cdot \mathrm{Al}_{2} \mathrm{O}_{3} \cdot \mathrm{CaSO}_{4} \cdot 12 \mathrm{H}_{22} \mathrm{O}
$$

o bien :

$$
\mathrm{CsH}_{2}+\mathrm{C}_{3} \mathrm{~A}+10 \mathrm{H} \rightarrow \quad \mathrm{C}_{3} \mathrm{ACsH}_{12}
$$

Esto llevaba a la conclusión de que, dada la riqueza de las materias primas españolas en alúmina, y la consiguiente abundancia de los cementos Portland en aluminato tricálcico, a estos cementos ha de corresponderles un "yeso óptimo" - o un $\mathrm{SO}_{3}$ "óptimo"- mayor que el correspondiente a otros cementos extranjeros más escasos en el citado aluminato.

Habida cuenta del contenido medio de alúmina de las materias primas nacionales, y del tanto por ciento correspondiente de $\mathrm{C}_{3} \mathrm{~A}$ potencial calculado para los cementos resultantes, que, según datos propios y ajenos (2) y (3), se elevaba - y se sigue elevando- al 13 ó 14, de la estequiometría de la ecuación [1] resulta que, en promedio, los clínkeres españoles precisan - o si se prefiere "toleran"- - una cantidad de yeso dihidrato, expresado en $\mathrm{SO}_{3}$, del $4 \%$ aproximadamente $-\mathrm{y}$ mayor aún, si en vez de yeso dihidrato se trata de anhidrita (4)-.

Estas ideas directrices, confirmadas plenamente por los resultados experimentales de los mencionados trabajos, llevaron a proponer, y condujeron a aceptar "némine discrepante" en el PCCH 61 (5), la elevación del límite máximo de $\mathrm{SO}_{3}$ hasta el $4 \%$. Por el carácter de máximo que esta limitación tiene, ello no podía nunca perjudicar a ningún clínker, puesto que los que por sus características no admitiesen una elevación en el contenido de yeso, sobre el valor que de ordinario tenían, podían quedar como hasta entonces. En cambio, la medida podía favorecer - como de hecho favoreció- a no pocos clínkeres que por su composición y constitución admitían -o "exigían" - más yeso para mejor desarrollar sus cualidades resistentes, de estabilidad de volumen y de durabilidad. Debe tenerse presente a este respecto, que cada clinker tiene su "yeso óptimo", según su composición y constitución, y que dicho óptimo puede oscilar algo, principalmente, en función de la finura del cemento resultante, todo lo cual debe constituir un motivo de vigilancia y control, si se quieren obtener del cemento las máximas posibilidades. En definitiva, la modificación del límite máximo de contenido de $\mathrm{SO}_{3}$ en los cementos, en la forma indicada, al no perjudicar en ningún caso y ser beneficiosa en muchos, constiluyó una medida justificada y oportuna. 
Posteriormente, con motivo de celebrarse en 1962 el I Coloquio Internacional sobre Obras Públicas en Terrenos Yesiferos, hubo ocasión de volver sobre el mismo tema y en los mismos términos, al tratar del yeso como constituyente de los conglomerantes hidráulicos (6). Allí se dio cuenta de la evolución que, en el sentido apuntado, experimentaron diversos países de los cinco Continentes, con posterioridad a la resolución adoptada de antemano y con carácter original en el nuestro.

La nueva y reciente versión de 1964 del Pliego de Condiciones para Conglomerantes Hidráulicos -- PCCH 64 (7) - ha mantenido el criterio aceptado en el PCCH 61, con ligeras modificaciones, que no sólo apoyan dicho criterio, sino que lo refuerzan considerablemente, como consecuencia de resultados experimentales más recientes, relativos a otros cementos que, si bien responden al tipo Portland, por sus características particulares entran en una clase especial —PAS, resistentes a agresivos selenitosos- (8). También en este caso y para análoga clase de cementos, varias Normas extranjeras han seguido, aunque con posterioridad, la misma línea de conducta, como habrá ocasión de ver detalladamente más adelante.

Finalmente, en los IV Coloquios de Directores y Técnicos de Fábricas de Cemento celebrados en 1964, y en una ponencia presentada sobre el tema Pliegos de Condiciones para los Cementos (9), se ha propuesto, en contraposición a todo lo anterior, "volver atrás", es decir, reducir el contenido máximo de $\mathrm{SO}_{3}$ aceptado y sancionado por el $\mathrm{PCCH}$ 64, a valores más en consonancia con los de otros países en general, y europeos en particular. La razón que se aducía para este retroceso es la de que, en una posible futura competencia internacional o europea de mercado, los cementos españoles dejarían de cumplir, en el aspecto de su contenido de $\mathrm{SO}_{3}$, con la mayoría de las Normas de otros países, viéndose por ello en inferioridad de condiciones competitivas. El razonamiento es inconsistente, por cuanto que el contenido de $\mathrm{SO}_{3}$ de los cementos según las normas es, prácticamente en la totalidad de los casos, un máximo que no obliga sino a no sobrepasarlo. $\mathrm{El}^{\mathrm{SO}_{3}}$ de los cementos puede potestativamente quedar por debajo y aun muy por debajo de dicho máximo, según cualesquiera conveniencias, pero, sobre todo, según lo que la composición y constitución del clínker y la finura exijan. $\mathrm{Ni}$ todos los cementos españoles deben, en razón a los clínkeres con que están hechos, alcanzar el máximo de $\mathrm{SO}_{3}$ admitido como posible por el pliego, ni hay por qué evitar que, los que por dicha razón puedan y deban alcanzarlo, lo alcancen. Y tanto más cuanto que son muchos los que se encuentran en este caso, circunstancia que no se da en la mayoría de los cementos extranjeros. Pero, con todo, aún cabría que estos cementos, de cara a una competencia internacional, se dosificaran con menos $\mathrm{SO}_{3}$ - pues, se repite, el carácter de máximo de la cifra del Pliego no lo impide-, aunque se daria entonces el hecho curioso de que estos cementos, por cumplir con normas extranjeras, serían, en todos los aspectos, de calidad inferior a la que tendrían si se limitasen a cumplir con el Pliego español. Por otra parte, si nuestro criterio antiguo sobre la conveniencia de elevar el contenido máximo permisible de $\mathrm{SO}_{3}$ de los cementos, y de mantener el actualmente establecido, ha encontrado eco en otros países en los que no concurren tantas razones como en el nuestro para ello, es prueba evidente de que está justificado. Así lo demuestran los datos aportados en dos de los trabajos citados antes (1) y (6), y, a mayor abundamiento, los que se aducen en lo que sigue, sin contar con los muy autorizados que en este mismo número pueden consultarse (4). 


\section{2--puesta al día del tema}

Lo que se expone a continuación tiene un valor confirmativo, reiterado y puesto al día, de cuanto con anterioridad se ha argumentado - con datos y argumentos propios y ajenos- en pro de la tesis sustentada. Se basa en la comparación de los datos relativos a los contenidos máximos de $\mathrm{SO}_{3}$ admitidos por las Normas para cemento de distintos países, según la publicación "Review of the Portland Cement Standards in the World", del CEMBUREAU, en sus versiones de 1955 y 1961, últimas aparecidas por el momento (10) y (11). Esta comparación permite establecer los resultados del Cuadro 1.

Según estos resultados, en los ocho primesos casos se observan seis aumentos, un desdoblamiento y dos nuevos establecimientos de valores máximos; en los tres últimos casos -en realidad son sólo dos- se aprecia una ligera disminución parcial.

Es de destacar el caso de Méjico, que establece distintos contenidos máximos de $\mathrm{SO}_{3}$ $(2,5,3,3,5$ y $4 \%$ ), el más bajo de ellos en función del cumplimiento dé unas condiciones respecto de álcalis, superficie específica y contenido de $\mathrm{C}_{3} \mathrm{~A}, \mathrm{y}$ los otros en función de los fallos (uno, dos o tres) al cumplimiento de dichas condiciones. Esto parece muy razonable. El valor de $4 \%$ de las actuales Normas españolas concuerda con el máximo de $4 \%$ de las Normas mejicanas, pues los cementos Portland españoles no cumplen, en general, con ninguna de las condiciones mínimas exigidas por las Normas mejicanas en cuanto a álcalis, superficie específica y contenido de $\mathrm{C}_{3} \mathrm{~A}$.

Interesa también destacar el hecho de que Méjico sea el único país que fije valores mínimos admisibles - “umbrales"- para el contenido de $\mathrm{SO}_{3}$ de los cementos, a saber, tos valores máximos en cada caso, disminuidos en un 1 por ciento.

Especial es el caso de Taiwan (China Nacionalista), que fija máximos de $\mathrm{SO}_{3}$ de 2 a 2,5 para contenidos de $\mathrm{C}_{3} \mathrm{~A}$ 'mayores de $8 \%$, y máximos de $\mathrm{SO}_{3}$ de 2,5 a 3 para contenidos de $\mathrm{C}_{3} \mathrm{~A}$ iguales o menores del $8 \%$. Es decir, los contenidos máximos de $\mathrm{SO}_{3}$ están en razón inversa de los contenidos de $\mathrm{C}_{3} \mathrm{~A}$. Esto parece ser un error de la publicación del CEMiBUREAU de 1961, porque las Normas de Taiwan se parecen mucho a las de U. S. A., en las que están inspiradas.

Como ampliación de lo expuesto en el Cuadro 2 se indican los tantos por ciento de $\mathrm{SO}_{3}$ -bien sea en forma de yeso dihidrato o de anhidrita-- que corresponden a los distintos tantos por ciento - de 1 a $20-$ de $\mathrm{C}_{33} \mathrm{~A}$ en el clinker, para su total transformación estequiométrica en $\mathrm{C}_{33} \mathrm{~A} \cdot \mathrm{Cs} \cdot \mathrm{H}_{12}$.

Por otra parte, la situación comparativa entre los años 1955 y 1961, respecto de los países cuyas Normas condicionan de un modo u otro el contenido máximo permisible de $\mathrm{SO}_{3}$ en los cementos al contenido - máximo o no-- de $\mathrm{C}_{3} \mathrm{~A}$ de los mismos, es la dada por los Cuadros 3.I, 3.II y 3.III.

De acuerdo con el Cuadro 2 y las indicacimes del Cuadro 3.III, los casos en que puede sobrar $\mathrm{SO}_{3}$, después de transformar todo el $\mathrm{C}_{3} \mathrm{~A}$ en $\mathrm{C}_{3} \mathrm{~A} \cdot \mathrm{Cs} \cdot \mathrm{H}_{12}$, se encuentran entre aquellos de las columnas $A$ en los cuadro 3.I y 3.II, cuyos valores son mayores que los correspondientes de las columnas $C$ de los mencionados cuadros. Estos casos están señalados en el vértice superior izquierdo de las respectivas casillas. Según el Cuadro 3.I, el número de estos casos en 1955 fue de 4 , sobre un total de 18, es decir, el $22 \%$; según el Cuadro 3.II, en 1961 fueron 12, de un total de 40, es decir, 30 por ciento. 
Los casos en que sobra $\mathrm{SO}_{3}$ después de transformar todo el $\mathrm{C}_{3} \mathrm{~A}$ en $\mathrm{C}_{3} \mathrm{~A} \cdot \mathrm{Cs} \cdot \mathrm{H}_{12}$ se encuentran entre aquéllos de las columnas $B$ en los Cuadros 3.I y 3.II, cuyos valores son mayores que los correspondientes de las columnas $C$ de los mencionados cuadros. Estos casos están señalados en el vértice inferior izquierdo de las respectivas casillas. Según el Cuadro 3.I, el número de estos casos en 1955 fue de 9, sobre un total de 18, es decir, $50 \%$; según el Cuadro 3.II, en 1961 fueron 22, de un total de 10, es decir, 55 por ciento.

Los casos en que puede faltar $\mathrm{SO}_{3}$ para transformar todo el $\mathrm{C}_{3} \mathrm{~A}$ en $\mathrm{C}_{3} \mathrm{~A} \cdot \mathrm{Cs} \cdot \mathrm{H}_{12}$ se encuentran entre aquéllos de las columnas $B$ en los Cuadros 3.I y 3.II, cuyos valores son menores que los correspondientes de las columnas $C$ de los mencionados cuadros. Estos casos están señalados en el vértice superior derecho de las respectivas casillas. Según el Cuadro 3.I, el número de estos casos en 1955 fue de 5, sobre un total de 18, es decir, $28 \%$; según el Cuadro 3.II, en 1961 fueron 3, de un total de 40, es decir, 7,5 por ciento.

Los casos en que falta $\mathrm{SO}_{3}$ para transformar todo el $\mathrm{C}_{3} \mathrm{~A}$ en $\mathrm{C}_{3} \mathrm{~A} \cdot \mathrm{Cs} \cdot \mathrm{H}_{12}$ se encuentran entre aquéllos de las columnas $A$ en los Cuadros 3.I y 3.II, cuyos valores son menores que los correspondientes de las columnas $C$ de los mencionados cuadros. Estos casos están señalados en el vértice inferior derecho de las respectivas casillas. Según el Cuadro 3.I, el número de estos casos en 1955 fue de 0 , sobre un total de 18 , es decir, $0 \%$; según el Cuadro 3.II, en 1961 fueron 3 , de un total de 40, es decir, 7,5 por ciento.

Estos resultados, así como las diferencias entre los mismos, se ponen de manifiesto en el Cuadro 4.I.

Los casos en que el $\mathrm{SO}_{3} \%$ es aproximadamente el necesario para transformar todo el $\mathrm{C}_{3} \mathrm{~A}$ en $\mathrm{C}_{3} \mathrm{~A} \cdot \mathrm{Cs} \cdot \mathrm{H}_{12}$, entendiéndose convencionalmente por tal el que difiere del $\mathrm{SO}_{3} \%$ teórico de las columnas $C$ de los cuadros 3.I y 3 .II en $\pm 0,3 \%$, son los que están señalados en el lado derecho o izquierdo de las respectivas casillas. Si se desglosan estos casos de las cuatro posibilidades consideradas en las columnas del Cuadro 4.I, hay que sustraer, en la primera columna, 2 casos en 1955 y 2 en 1961; en la segunda, 6 y 10; en la tercera, 3 y 1 ; en la cuarta, ninguno en ambos casos. El total de los casos sustraídos es de 11 en 1955 y 13 en 1961, quedando el Cuadro 4.I convertido en el 4.IT.

Si se tiene en cuenta la posible - casi segura - inversión de los datos correspondientes a Taiwan en el Cuadro 3.II, en el sentido de que lógicamente deben intercambiarse las columnas $e \mathrm{y} j$, los Cuadros 4.I y 4.II quedan convertidos en los 4.III y 4.IV.

De las diferencias señaladas en cualquiera de estos cuadros, y particularmente de las del Cuadro 4.IV, se deduce fảcilmente que entre 1955 y 1961 ha existido una bien marcada tendencia a aumentar el número de casos en que puede sobrar, o de hecho sobra en los cementos $\mathrm{SO}_{3}$ para transformar todo el $\mathrm{C}_{3} \mathrm{~A}$ de los mismos en $\mathrm{C}_{3} \mathrm{~A} \cdot \mathrm{Cs}_{\mathrm{S}} \cdot \mathrm{H}_{12}$. Esto indica, a su vez, que los tantos por ciento de retardadores sulfatados añadidos a los cementos han aumentado en dicho período de tiempo, lo que confirma, en otros casos, y por otros caminos, lo expresado en el Cuadro $1 \mathrm{y}$ deducido del mismo.

Esta tendencia se ha manifestado a costa de los casos en que el tanto por ciento de $\mathrm{SO}_{3}$ en los cementos podía considerarse aproximadamente - con una diferencia máxima de $\pm 0,3 \%$ - como el necesario --teórico-para transformar en $\mathrm{C}_{3} \mathrm{~A} \cdot \mathrm{Cs} \cdot \mathrm{H}_{12}$ todo el $\mathrm{C}_{3} \mathrm{~A}$ de los mismos.

No es ese el caso de España, pues el máximo actual de $\mathrm{SO}_{3} \%$ cubre los cementos hasta con 13-14 \% de $\mathrm{C}_{3} \mathrm{~A}$, siendo de advertir que el límite superior del $\mathrm{C}_{3} \mathrm{~A}$ en los cementos 
Portland no especialmente resistentes a sulfatos se sitúa en $18 \%$, a lo que correspondería $5,3 \%$ de $\mathrm{SO}_{3}$ máximo para la transformación total del $\mathrm{C}_{3} \mathrm{~A}$ en $\mathrm{C}_{3} \mathrm{~A} \cdot \mathrm{Cs} \cdot \mathrm{H}_{12}$.

Con los cementos resistentes a los agresivos selenitosos en mayor o menor medida, la situación en 1955 y 1961, según los datos publicados por el CEMBUREAU, es la presentada en los Cuadros 5.I y 5.II. Las diferencias $N$ - $t$ entre los valores $N$ de $\mathrm{SO}_{3}$ máximos según las Normas, y los valores $t$ de $\mathrm{SO}_{3}$ máximos teóricos para transformar los contenidos máximos de $\mathrm{C}_{3} \mathrm{~A} \%$ según las Normas en $\mathrm{C}_{3} \mathrm{~A} \cdot \mathrm{Cs} \cdot \mathrm{H}_{12}$, están indicadas en el Cuadro 5:IIII. En él están calculadas las medias de dichas diferencias en los años 1955 y 1961. La media de las diferencias positivas $(N>t)$ es mayor - del orden del doble- en 1961 que en 1955; la media de las diferencias negativas $(N<t)$ es del mismo orden en ambos años. Las sumas algébricas de las medias de dichas diferencias son positivas en ambos años, y mayor - del orden del doble - la de 1961 que la de 1955.

Todo esto indica que también para los cementos resistentes a los agresivos selenitosos se ha manifestado claramente entre 1955 y 1961 una tendencia a aumentar el número de casos en que puede sobrar, o de hecho sobra, en dichos cementos, $\mathrm{SO}_{3}$ para transformar todo el $\mathrm{C}_{3} \mathrm{~A}$ de los mismos en $\mathrm{C}_{3} \mathrm{~A} \cdot \mathrm{Cs} \cdot \mathrm{H}_{12}$. Ello indica también que los tantos por ciento de retardadores sulfatados añadidos a los cementos resistentes a los ataques selenitosos han aumentado en dicho período de tiempo, lo que confirma una vez más lo expresado en el Cuadro $1 \mathrm{y}$ deducido del mismo. Esto justifica, al propio tiempo, el haber considerado en los Cuadros 3.I y 3.II, y por lo tanto, también en los Cuadros 4.I, 4.II, 4.III y 4.IV, los cementos resistentes a los agresivos selenitosos englobados con los restantes cementos Portland que' no poseen especialmente dicha característica.

Por todos estos hechos, la reciente Norma española para cementos, en su versión de 1964, ha considerado oportuno elevar también a $4 \%$ el contenido máximo de $\mathrm{SO}_{3}$ de los cementos Portland resistentes a los agresivos selenitosos, aun cuando en estos cementos el contenido máximo admisible de $\mathrm{C}_{3} \mathrm{~A}$ sea de $5 \%$. A este valor correspondería $1,5 \%$ teórico de $\mathrm{SO}_{3}$ para su transformación total en $\mathrm{C}_{3} \mathrm{~A} \cdot \mathrm{Cs} \cdot \mathrm{H}_{12}$. No obstante, la decisión adoptada se apoya también en hechos y resultados experimentales, según los cuales los cementos Portland resistentes a los ataques selenitosos, con bajo contenido de $\mathrm{C}_{33} \mathrm{~A}$, mejoran igualmente en cuanto a resistencias mecánicas y estabilidad, cuando su contenido de yeso se eleva discretamente sobre el valor que usualmente suele tener (8).

Parece como si en los cementos Portland normales - españoles- con relaciones $\mathrm{C}_{3} \mathrm{~A}$ / $\mathrm{C}_{4} \mathrm{AF}$ del orden de 2 en promedio $\left(\mathrm{C}_{3} \mathrm{~A}=14 \% ; \mathrm{C}_{44} \mathrm{AF}=7 \%\right)$, variables entre $1,5\left(\mathrm{C}_{3} \mathrm{~A}=\right.$ $\left.=12 \% ; \mathrm{C}_{44} \mathrm{AF}=8 \%\right)$ y $2,5\left(\mathrm{C}_{3} \mathrm{~A}=15 \% ; \mathrm{C}_{4} \mathrm{AF}=6 \%\right)$, fuese el $\mathrm{C}_{33} \mathrm{~A}$ el factor determinante de la reacción del retardador sulfatado, mientras que en los cementos Portland resistentes a los agresivos selenitosos, con relaciones $\mathrm{C}_{3} \mathrm{~A} / \mathrm{C}_{4} \mathrm{AF}$ del orden de 0,25 en promedio $\left(\mathrm{C}_{3} \mathrm{~A}=5 \% ; \mathrm{C}_{4} \mathrm{AF}=20 \%\right)$, variables entre $0,10\left(\mathrm{C}_{3} \mathrm{~A}=2 \% ; \mathrm{C}_{4} \mathrm{AF}=20 \%\right)$ y $0,3\left(\mathrm{C}_{3} \mathrm{~A}=5 \% ; \mathrm{C}_{4} \mathrm{AF}=14 \%\right)$ el factor determinante de la reacción del retardador fuese el $\mathrm{C}_{4} \mathrm{AF}$, o más bien alguna de las disoluciones sólidas ferríticas que en tales condiciones pueden existir.

Esta última consideración parece suscitar el interés que tendría el conocer la interacción entre los retardadores sulfatados y las fases ferríticas de los cementos Portland, tanto normales como escasos de alúmina y ricos en óxido férrico. 


\section{3. - aspectos particulares}

Como puede apreciarse, en lo anterior se ha hecho referencia a los cementos de tipo Portland, resistentes o no a los agresivos selenitosos. Si con arreglo a la definición de Portland del Pliego español se consideran como tales aquellos conglomerantes que contienen hasta un $10 \%$ de materias distintas del Portland, en dichos cementos $\rightarrow \mathrm{y}$ mucho más aún en los de adición (tipo C 150), o en los que por contener adiciones en dosis superiores al $10 \%$ quedan fuera del Pliego- hay que tener presente y debe sobreentenderse que para no caer perniciosamente en el extremo opuesto, la dosificación en tanto por ciento de retardador sulfatado - yeso, anhidrita o mezcla - expresada en $\mathrm{SO}_{3}$, debe calcularse sobre el clínker de cemento Portland que contenga el "conglomerante", y no sobre el total de éste, pues, en el último caso, el "conglomerante" seria una mezcla -en la proporción que fuese- de una adición y de un cemento excesivamente sobredosificado de retardador, con peores características de todo tipo, y de utilización peligrosa.

Por razones de tipo análogo, los conglomerantes de tipo distinto al Portland y constituidos por clínker y adiciones activas --escorias o puzolanas- deberán contener dosis de retardadores sulfatados en armonía con la composición, constitución y proporción, tanto del clínker como de las citadas adiciones activas, así como con la finura del conglomerante que se quiera obtener (4).

Un aspecto especialmente interesante, aparte de los científico-técnicos ya expuestos, es el económico. El paso de $2,5 \%$ máximo de $\mathrm{SO}_{3}$ a $4 \%$ en los cementos de tipo Portland - prácticamente en la totalidad de la producción española de cemento- podia suponer, para la producción de 1961 - unos 6 millones de toneladas - un aumento libre de gastos por todos conceptos de 150 a 200 mil toneladas anuales; para la producción de 1962 - unos 6,5 millones de toneladas-, de 165 a 210 mil toneladas; para la de 1964 -unos 8 millones de toneladas - de 200 a 260 mil toneladas; y para la actual - unos 9 millones de toneladas anuales- de 230 a 290 mil toneladas. En cualquier caso, estos aumentos supondrian del 2,5 al 3,5 - en promedio el 3 - por ciento de la producción. Parte de estos datos ya fueron dados a conocer en su día (1), (6) y (12).

\section{4. - conclusiones}

El resumen de las conclusiones sobre el tema del contenido de retardadores sulfatados en los cementos, es el siguiente:

1. a En el caso de los cementos Portland, cada clínker requiere un contenido "óptimo" de retardador - yeso dihidrato, anhidrita o mezcla de ambos-para dar lugar a un cemento "correctamente retardado" y de mejores cas acterísticas mecảnicas, de estabilidad de volumen y de durabilidad. Este contenido óptimo depende de la composición y constitución química y mineralógica del clínker fundamentalmente, a igualdad de otros factores de tipo físico - finura y granulometría del cemento como más importantes--. Entre los aspectos quimicos y mineralógicos más influyentes destacan el contenido de aluminato trícálcico - eventualmente de fase ferrítica-, su estado vítreo o cristalino - dependiente de la 
velocidad de enfriamiento del clínker-, el contenido de álcalis y la forma en que éstos se encuentran - lo que depende de las materias primas y del proceso de fabricación-.

2. ${ }^{a} \quad$ El contenido óptimo de retardador sulfatado, variable entre ciertos límites según lo expuesto en la conclusión anterior, corresponde aproximadamente, en el promedio de los casos, a la cantidad precisa para transformar el aluminato tricálcico potencial del cemento en sulfoaluminato cálcico monosulfato hidratado. Se eleva algo con el contenido de álcalis, con la finura y con el estado vítreo cristalino del aluminato tricálcico, así como con la condición anhidra del retardador - anhidrita--. El tanto por ciento óptimo de retardador, expresado como $\mathrm{SO}_{3}$, y habida cuenta de las características medias de los clínkeres españoles, según las materias primas empleadas, se cifra, en promedio, en 4 . Este valor es necesariamente superior al correspondiente en la casi totalidad de las Normas para cemento de los demás países, por cuanto que en casi ninguno de ellos se dan materias primas y crudos tan aluminosos, o clínkeres tan ricos en aluminato tricálcico $-\mathrm{y}$ eventualmente en álcalis-. Esto no quiere decir que no haya cementos cuyo óptimo de retardador rebase el $4 \%$ de $\mathrm{SO}_{3}$, puesto que hay clínkeres - y así lo prevén los Pliegos $\mathrm{PCCH} 61$ y 64 - que pueden contener hasta el $18 \%$ de $\mathrm{C}_{3} \mathrm{~A}$, siendo así que dicho $4 \%$ de $\mathrm{SO}_{3}$ corresponde a un contenido de $\mathrm{C}_{3} \mathrm{~A}$ del $14 \%$ aproximadamente, según la estequiometría de la ecuación [I].

3. ${ }^{a}$ En el caso de los cementos Portland resistentes a los agresivos selenitosos también se ha observado una mejora en todas sus propiedades, cuando se eleva en una cierta medida su contenido de retardador sulfatado. Será necesario estudiar el caso de estos conglomerantes y comprobar si, como parece, las fases determinantes en su caso de la reacción con el retardador sulfatado son las ferríticas, a diferencia de lo que sucede en los Portland ordinarios, en los que parece ser principalmente el $\mathrm{C}_{3} \mathrm{~A}$ - ecuación [I $]$ - .

4. ${ }^{\mathrm{a}} \quad$ No existe ninguna razón, mientras no se demuestre con hechos incontrovertibles lo contrario, para no mantener en el PCCH español el valor máximo admisible de $4 \%$ de $\mathrm{SO}_{3}$, indicado en la conclusión $2 .^{a}$ para los retardadores sulfatados. Su carácter de máximo susceptible de ser potestativamente alcanzado o no, invalida cualquier argumento en pro de todo intento de rebajar dicho valor, al amparo de una forzada e improcedente aproximación a las exigencias de las Normas extranjeras, injustificable de todo punto por razones científico-técnicas. Si estas Normas extranjeras no llegan a alcanzar el valor admitido en la española es, ante todo, porque no lo exigen las características de sus materias primas, crudos y clínkeres. Pese a ello, la tendencia creciente de muchas de dichas Normas en los últimos diez años es a elevar, cada vez más, sus límites máximos de $\mathrm{SO}_{3}$, a semejanza de lo hecho en nuestro país con anterioridad, y por motivos suficientemente justificados y explicados, y sancionados, por añadidura, con hechos experimentales unánimemente concordantes, aun procediendo de distintos laboratorios.

$5^{\text {a }}$ Debería prestarse la máxima atención a la dosificación óptima de retardador sulfatado, en el caso de los cementos de adiciones activas - siderúrgicos y puzolánicos - y muy especialmente en los cementos llamados de adición, o que contengan adiciones inertes. En este último caso debe calcularse la dosificacón del retardador de acuerdo con la naturaleza y proporción del clinker en dichos conglomerantes. 
CUADRO 1

\begin{tabular}{|c|c|c|}
\hline & \multicolumn{2}{|c|}{$\begin{array}{c}\text { Datos que figuran en "Review of the Portland } \\
\text { Cement Standards of the World", } \\
\text { del CEMBUREAU. }\end{array}$} \\
\hline & \multicolumn{2}{|c|}{$\mathrm{SO}_{3}$ máx. $\%$} \\
\hline & 1955 & 1961 \\
\hline Bélgica & 3 & 3,75 \\
\hline China & 2,5 & 3 \\
\hline Francia & 3 & 3,5 \\
\hline Hungría & - & 3 \\
\hline Portugal & $2-2,5$ & 3 \\
\hline ESPAÑA & 2,5 & 4 \\
\hline Suecia & - & 3,5 \\
\hline Africa del Sur & 2,75 & 3 \\
\hline Estados Unidos & 2,3 (tipo V) & 3 (tipo V) \\
\hline Inglaterra & \multirow{2}{*}{2,75} & 2,5 \\
\hline Nueva Zelanda & & 2,10 \\
\hline Rumania & 3 & 3 \\
\hline
\end{tabular}

CUADRO 2

\begin{tabular}{|c|c|}
\hline $\mathrm{C}_{3} \mathbf{A} \%$ & $\begin{array}{c}\mathrm{SO}_{3} \% \\
\text { para formar } \mathrm{C}_{3} \text { A. Cs. } \mathrm{H}_{12}\end{array}$ \\
\hline $\begin{array}{r}1 \\
2 \\
3 \\
4 \\
5 \\
6 \\
7 \\
8 \\
9 \\
10 \\
11 \\
12 \\
13 \\
14 \\
15 \\
16 \\
17 \\
18 \\
19 \\
20\end{array}$ & $\begin{array}{l}0,3 \\
0,6 \\
0,9 \\
1.2 \\
1,5 \\
1,8 \\
2,1 \\
2,4 \\
2,7 \\
3,0 \\
3,3 \\
3,6 \\
3,9 \\
4.1 \\
4,4 \\
4,7 \\
5,0 \\
53 \\
5,6 \\
5,9\end{array}$ \\
\hline
\end{tabular}


CUADRO 3.1

CEMBUREAU 1955

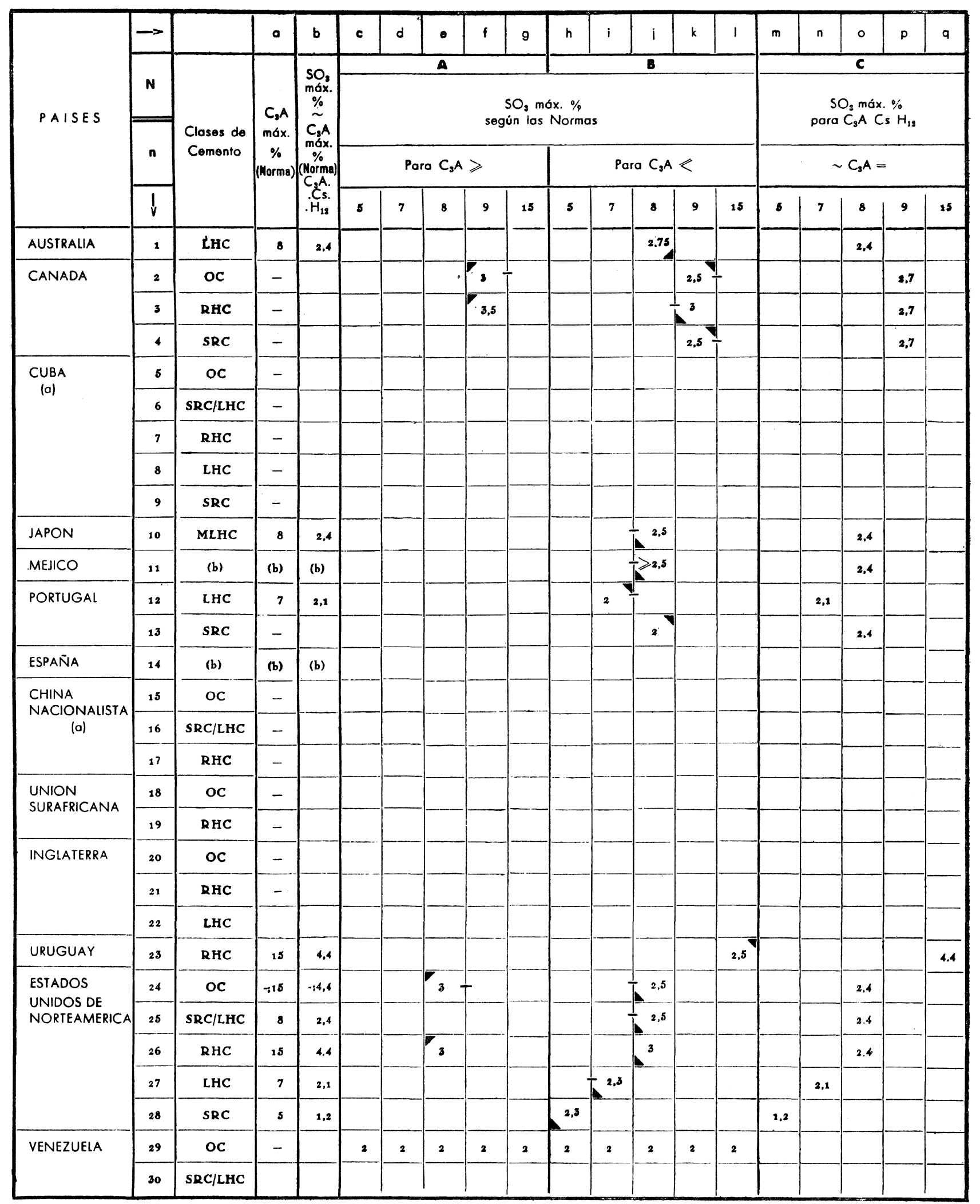


CUADRO 3.11

CEMBUREAU 1951

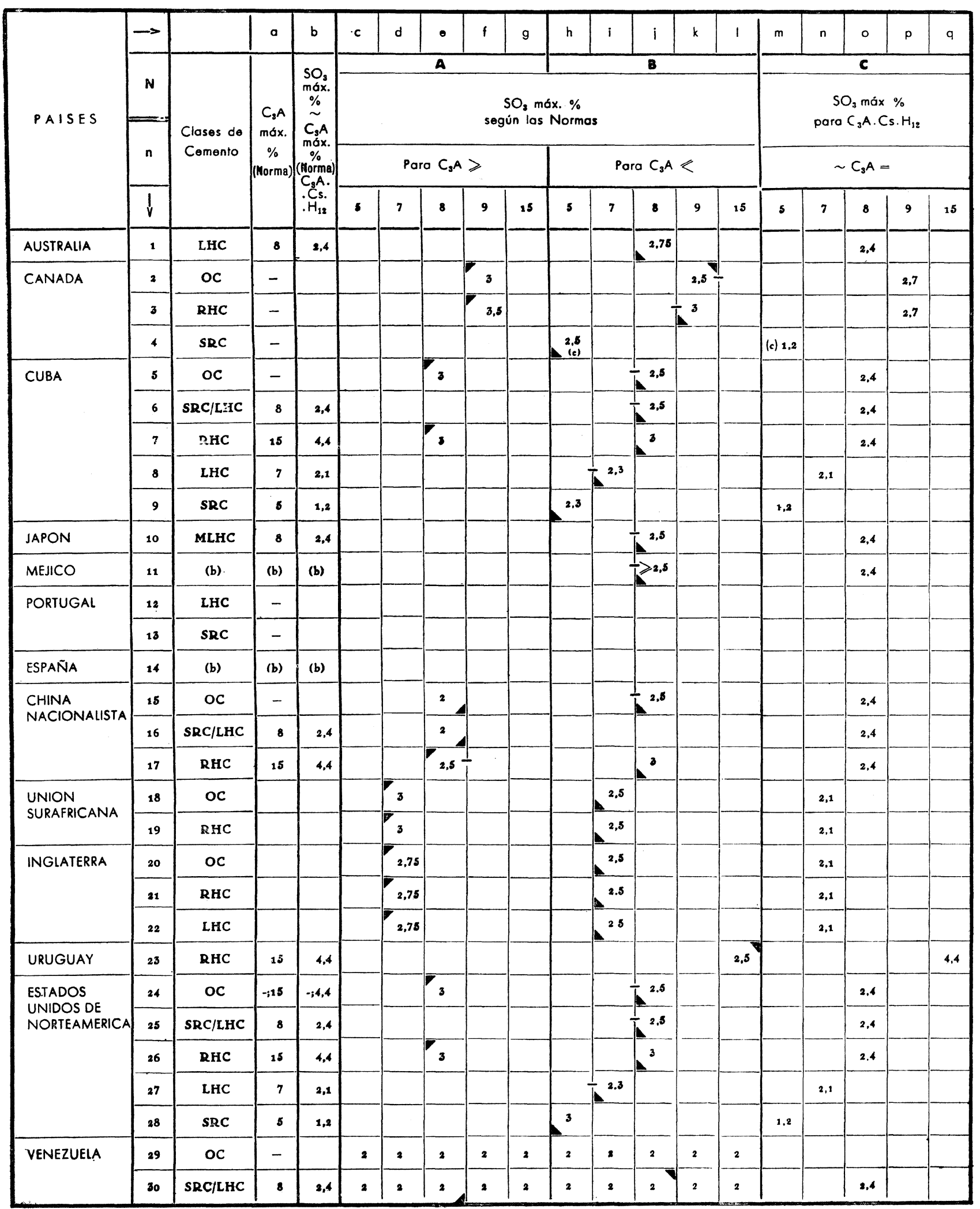


CUADRO 3. III

Aclaraciones a los cuadros $3 \mid$ y 3 II

OC = Ordinary Cement (Cemento Portlant corriente).

RHC = Rapid Hardening Cement (Cemento Portland de Eudurecimiento Rápido).

$\mathrm{SRC}=$ Sulphate Resistent Cement (Cemento Porlland Resistente a los Sulfatos).

LHC = Low Heat Cement (Cemento Portland de Bajo calor de Hidratación).

MLHC $=$ Moderate Low Heat Cement (Cemento Portland de Moderado calor de Hidrata. ción).

(a) No figura en los datos del CEMBUREAU 1955;

(b) Detalles en el texto;

(c) Corresponde a $\mathrm{C}_{3} \mathrm{~A}<4 \%$ en vez de a $\mathrm{C}_{3} \mathrm{~A}<5 \%$;

casos en que puede sobrar $\mathrm{SO}_{3}$ después de transformar todo el $\mathrm{C}_{3} \mathrm{~A}$ en $\mathrm{C}_{3} \mathrm{~A}$. - $\mathrm{Cs} \cdot \mathrm{H}_{12}$;

Casos en que sobra $\mathrm{SO}_{3}$ después de transformar todo el $\mathrm{C}_{3} \mathrm{~A}$ en $\mathrm{C}_{3} \mathrm{~A} \cdot \mathrm{Cs} \cdot \mathrm{H}_{12}$;

Casos en que puede faltar $\mathrm{SO}_{3}$ para transformar todo el $\mathrm{C}_{3} \mathrm{~A}$ en $\mathrm{C}_{3} \mathrm{~A} \cdot \mathrm{Cs} \cdot \mathrm{H}_{12}$;

Casos en que falta $\mathrm{SO}_{3}$ para transformar todo el $\mathrm{C}_{3} \mathrm{~A}$ en $\mathrm{C}_{3} \mathrm{~A} \cdot \mathrm{Cs} \cdot \mathrm{H}_{12}$.

1

Casos en que el $\mathrm{SO}_{3} \%$ es el necesario $( \pm 0,3 \%)$ para transformar todo el $\mathrm{C}_{3} \mathrm{~A}$ en $\mathrm{C}_{10} \mathrm{~A} \cdot \mathrm{Cs} \cdot \mathrm{H}_{12}$. 
CUADRO 4. 1

\begin{tabular}{|c|c|c|c|c|c|c|c|c|c|c|}
\hline \multirow{2}{*}{\multicolumn{2}{|c|}{$\begin{array}{l}\text { Número de casos } n \\
\text { y } \% \text { de casos }\end{array}$}} & \multicolumn{9}{|c|}{$\mathrm{SO}_{3} \%$ para $\mathrm{C}_{3}$ A. Cs. $\mathrm{H}_{12}$} \\
\hline & & \multicolumn{2}{|c|}{$\begin{array}{l}\text { Puede } \\
\text { sobrar }\end{array}$} & \multicolumn{2}{|c|}{ Sobra } & \multicolumn{2}{|c|}{$\begin{array}{l}\text { Puede } \\
\text { faltar }\end{array}$} & \multicolumn{2}{|c|}{ Falta } & \multirow{2}{*}{$\frac{\text { Total }}{18}$} \\
\hline \multirow{2}{*}{1955} & $n$ & 4 & & 9 & & 5 & & 0 & & \\
\hline & $\%$ & & 22 & & 50 & & 28 & & 0 & 100 \\
\hline \multirow{2}{*}{1961} & $n$ & 12 & & 22 & & 3 & & 3 & & 40 \\
\hline & $\%$ & & 30 & & 55 & & 7,5 & & 7,5 & 100 \\
\hline \multirow{2}{*}{$\begin{array}{c}\text { Diferencias } \\
1961-1955\end{array}$} & $n$ & +8 & & +13 & & -2 & & +3 & & - \\
\hline & $\%$ & & +8 & & +5 & & $-20,5$ & & $+7,5$ & - \\
\hline
\end{tabular}

CUADRO 4.II

\begin{tabular}{|c|c|c|c|c|c|c|c|c|c|c|c|c|}
\hline \multirow{2}{*}{\multicolumn{2}{|c|}{$\begin{array}{l}\text { Número de casos } n \\
\text { y } \% \text { de casos }\end{array}$}} & \multicolumn{11}{|c|}{$\mathrm{SO}_{3}$ para $\mathrm{C}_{3} \mathrm{~A} . \mathrm{Cs} . \mathrm{H}_{12}$} \\
\hline & & \multicolumn{2}{|c|}{$\begin{array}{l}\text { Puede } \\
\text { sobrar }\end{array}$} & \multicolumn{2}{|c|}{ Sobra } & \multicolumn{2}{|c|}{$\begin{array}{l}\text { Puede } \\
\text { faltar }\end{array}$} & \multicolumn{2}{|c|}{ Falta } & \multicolumn{2}{|c|}{$\begin{array}{c}\text { Hay lo justo } \\
( \pm 0,3 \%)\end{array}$} & \multirow{2}{*}{$\frac{\text { Total }}{18}$} \\
\hline \multirow{2}{*}{1955} & $n$ & 2 & & 3 & & 2 & & 0 & & 11 & & \\
\hline & $\%$ & & 11 & & 17 & & 11 & & 0 & & 61 & 100 \\
\hline \multirow{2}{*}{1961} & $n$ & 10 & & 12 & & 2 & & 3 & & 13 & & 40 \\
\hline & $\%$ & & 25 & & 30 & & 5 & & 7,5 & & 32,5 & 100 \\
\hline Diferencias & $n$ & +8 & & +9 & & 0 & & +3 & & +2 & & - \\
\hline $1961-1955$ & $\%$ & & +14 & & +13 & & -6 & & $+7,5$ & & $-28,5$ & - \\
\hline
\end{tabular}


CUADRO 4. III

\begin{tabular}{|c|c|c|c|c|c|c|c|c|c|c|}
\hline \multirow{2}{*}{\multicolumn{2}{|c|}{$\begin{array}{c}\text { Número de casos } n \\
\text { y } \% \text { de cosos }\end{array}$}} & \multicolumn{9}{|c|}{$\mathrm{SO}_{3} \%$ para $C_{3}$ A. Cs. $\mathrm{H}_{12}$} \\
\hline & & \multicolumn{2}{|c|}{$\begin{array}{l}\text { Puede } \\
\text { sobrar }\end{array}$} & \multicolumn{2}{|c|}{ Sobra } & \multicolumn{2}{|c|}{$\begin{array}{l}\text { Puede } \\
\text { faltar }\end{array}$} & \multicolumn{2}{|c|}{ Falta } & \multirow{2}{*}{$\frac{\text { Total }}{18}$} \\
\hline \multirow{2}{*}{1955} & $n$ & 4 & & 9 & & 5 & & 0 & & \\
\hline & $\%$ & & 22 & & 50 & & 28 & & 0 & 100 \\
\hline \multirow{2}{*}{1961} & $n$ & 13 & & 21 & & 5 & & 1 & & 40 \\
\hline & $\%$ & & 32,5 & & 52,5 & & 12,5 & & 2,5 & 100 \\
\hline \multirow{2}{*}{$\begin{array}{c}\text { Diferencias } \\
1961 \cdot 1955\end{array}$} & $n$ & +9 & & +12 & & 0 & & +1 & & - \\
\hline & $\%$ & & $+10,5$ & & $+2,5$ & & $-15,5$ & & $+2,5$ & - \\
\hline
\end{tabular}

CUADRO 4.IV

\begin{tabular}{|c|c|c|c|c|c|c|c|c|c|c|c|c|}
\hline \multirow{2}{*}{\multicolumn{2}{|c|}{$\begin{array}{l}\text { Número de casos n } \\
\text { y } \% \text { de casos }\end{array}$}} & \multicolumn{11}{|c|}{$\mathrm{SO}_{3}$ para $\mathrm{C}_{3} \mathrm{~A}$. Cs. $\mathrm{H}_{12}$} \\
\hline & & \multicolumn{2}{|c|}{$\begin{array}{l}\text { Puede } \\
\text { sobrar }\end{array}$} & \multicolumn{2}{|c|}{ Sobra } & \multicolumn{2}{|c|}{$\begin{array}{l}\text { Puede } \\
\text { faltar }\end{array}$} & \multicolumn{2}{|c|}{ Falta } & \multicolumn{2}{|c|}{$\begin{array}{c}\text { Hay lo justo } \\
( \pm 0,3 \%)\end{array}$} & \multirow{2}{*}{$\frac{\text { Total }}{18}$} \\
\hline & $n$ & 2 & & 3 & & 2 & & 0 & & 11 & & \\
\hline & $\%$ & & 11 & & 17 & & 11 & & 0 & & 61 & 100 \\
\hline \multirow{2}{*}{1961} & $n$ & 11 & & 11 & & 4 & & 1 & & 13 & & 40 \\
\hline & $\%$ & & 27,5 & & 27,5 & & 10 & & 2,5 & & 32,5 & 100 \\
\hline Diferencias & n & 9 & & +8 & & +2 & & +1 & & +2 & & - \\
\hline $1961-1955$ & $\%$ & & $+16,5$ & & $+10,5$ & & -1 & & +2.5 & & $-28,5$ & - \\
\hline
\end{tabular}


CUADRO 5.1

\begin{tabular}{|c|c|c|c|c|c|c|c|c|c|c|}
\hline \multirow{4}{*}{ PAISES } & \multirow{4}{*}{$\begin{array}{c}\text { Coordenadas } \\
\text { del } \\
\text { cuadro 3.I }\end{array}$} & \multicolumn{9}{|c|}{ Cementos SRC 1955} \\
\hline & & \multicolumn{9}{|c|}{$\mathrm{SO}_{3}$ máx. \% } \\
\hline & & \multicolumn{3}{|c|}{$N>t$} & \multicolumn{3}{|c|}{$\mathrm{N}<\mathrm{t}$} & \multicolumn{3}{|c|}{$\mathbf{N} \sim=\mathrm{t}$} \\
\hline & & $\mathbf{N}$ & $\mathbf{t}$ & Dif. & $\mathbf{N}$ & $t$ & Dif. & $\mathbf{N}$ & $t$ & Dif. \\
\hline Canadá & $\begin{array}{l}4 k \\
4 p\end{array}$ & & & & 2,5 & 2,7 & $-0,2$ & & & \\
\hline Portugal & $\begin{array}{l}13 i \\
130\end{array}$ & & & & 2 & 2,4 & $-0,4$ & & & \\
\hline \multirow[t]{2}{*}{$\begin{array}{l}\text { Estados Unidos de } \\
\text { Norteamérica }\end{array}$} & $\begin{array}{l}25 i \\
250\end{array}$ & & & & & & & 2,5 & 2,4 & $+0,1$ \\
\hline & $\begin{array}{l}28 \mathrm{~h} \\
28 \mathrm{~m}\end{array}$ & 2,3 & 1,2 & $+1,1$ & & & & & & \\
\hline
\end{tabular}

$\mathrm{N}: \mathbf{S}_{3} \mathrm{O}$ máx \% fijado por las Normas

$t$ : $\mathrm{SO}_{3}$ máx. $\%$ correspondiente al $\mathrm{C}_{3} \mathrm{~A}$ máx. $\%$ para formar $\mathrm{C}_{3} \mathrm{~A}$. Cs. $\mathrm{H}_{12}$

CUADRO 5. II

\begin{tabular}{|c|c|c|c|c|c|c|c|c|c|c|}
\hline \multirow{4}{*}{ PAISES } & \multirow{4}{*}{$\begin{array}{c}\text { Coordenadas } \\
\text { del } \\
\text { cuadro 3.I }\end{array}$} & \multicolumn{9}{|c|}{ Cementos SRC 1961} \\
\hline & & \multicolumn{9}{|c|}{$\mathbf{S O}_{3}$ máx. $\%$} \\
\hline & & \multicolumn{3}{|c|}{$N>t$} & \multicolumn{3}{|c|}{$\mathbf{N}<\mathrm{t}$} & \multicolumn{3}{|c|}{$\mathbf{N} \sim=\mathbf{t}$} \\
\hline & & $\mathbf{N}$ & $\mathrm{t}$ & Dif. & $N$ & $t$ & Dif. & $N$ & $t$ & Dif. \\
\hline Canadá & $\begin{array}{l}4 \mathrm{~h} \\
4 \mathrm{~m}\end{array}$ & 2,5 & 1,2 & $+1,3$ & & & & & & \\
\hline Cuba & $\begin{array}{l}9 \mathrm{~h} \\
9 \mathrm{~m}\end{array}$ & 2,3 & 1,2 & $+1,1$ & & & & & & \\
\hline China Nacionalista & $\begin{array}{l}16 e, i \\
160\end{array}$ & & & & 2 & 2,4 & $-0,4$ & & & \\
\hline \multirow[t]{2}{*}{$\begin{array}{l}\text { Estados Unidos de } \\
\text { Norteamérica }\end{array}$} & $\begin{array}{l}28 \mathrm{~h} \\
28 \mathrm{n}\end{array}$ & 3 & 1,2 & $+1,8$ & & & & & & \\
\hline & $\begin{array}{l}25 i \\
250\end{array}$ & & & & & & & 2,5 & 2,4 & $+0,1$ \\
\hline Venezuela & $\begin{array}{l}30 e, i \\
30 \circ\end{array}$ & & & & 2 & 2,4 & $-0,4$ & & & \\
\hline
\end{tabular}

$\mathrm{N}: \mathrm{SO}_{3}$ máx. \% fijado por las Normas

$\mathbf{t}: \mathrm{SO}_{3}$ máx. \% correspondiente al $\mathrm{C}_{3} \mathrm{~A}$ máx. \% para formar $\mathrm{C}_{3} \mathrm{~A}$. Cs. $\mathrm{H}_{12}$ 
CUADRO 5. III

\begin{tabular}{|c|c|c|c|}
\hline \multirow{4}{*}{ Años } & \multicolumn{3}{|c|}{ Cementos SRC } \\
\hline & \multicolumn{3}{|c|}{$\mathrm{SO}_{3}$ máx. \% } \\
\hline & \multicolumn{3}{|c|}{ Diferencias $N-t$} \\
\hline & Positivas (a) & Negativas (b) & $a+b$ \\
\hline 1955 & $\begin{array}{r}+1,1 \\
+0,1\end{array}$ & $\begin{array}{l}-0,2 \\
-0,4\end{array}$ & \\
\hline Medias & $+0,6$ & $-0,3$ & $+0,3$ \\
\hline 1961 & $\begin{array}{r}+1,3 \\
+1,1 \\
+1,8 \\
+0,1\end{array}$ & $\begin{array}{l}-0,4 \\
-0,4\end{array}$ & \\
\hline Medias & +1.1 & -04 & +0.7 \\
\hline
\end{tabular}

\section{referemcias}

1. J. Calleita: "Influencia del contenido en yeso del cemento Portland sobre la retracción de pastas, morteros y hormigones". Trabajos de la III Reunión Internacional sobre Reactividad de los Sólidos, 3 (V), 213-246. Madrid, 1956.

2. D. Guinea: Discusión en: J. M. Balaguer: "Métodos y procedimientos de ensayo: Pliego de Condiciones”. I. E. T. C. C. Publicación 15\%. Madrid, 1954.

3. S. Gunnea: Comunicación privada, 1955.

4. E. Schwiete y U. Ludwig: "Sobre la utilización de diferentes compuestos sulfatados en la obtención de cemento". U. A. M. C. (I. E. T. C. C.), núm. 120. Madrid, 1965.

5. P.C.C.H. 61: "Pliego de Condiciones para la Recepción de Conglomerantes Hidráulicos". Normas y Manuales. I. E. T. C. C. Madrid, 1961.

6. J. Calleja: "El yeso como constituyente de los conglomerantes hidráulicos". I Congreso Internacional sobre Obras Públicas en Terrenos Yesiferos. Madrid, 1962.

7. P.C.C.H. 64: “Pliego de Condiciones para la Recepción de Conglomerantes Hidráulicos". Normas y Manuales. I. E. T. C. C. Madrid, 1964.

8. F. Soria: "Adiciones de yeso y anhidrita a los cementos PAS (Portland resistentes a agresivos selenitosos)". Comunicación privada, 1965.

9. C. DE Benito: "Pliegos actuales, Pliegos generales y Pliegos especiales. IV Coloquios de Directores y Técnicos de Fábricas de Cemento. Madrid, 1964. T. A. M. C. (I. E. T. C. C.), núm. 118-119. Madrid, 1965

10. Cembureau Papers: "Review of the Portland Cement Standards of the World, 1955". Malmö, Suecia, 1956 .

11. Crmburan Papers: "Review of the Portland Cement Standards of the World, 1961". Malmö, Suecia, 1962.

12. J. Calleja: "Mejora de la productividad en las industrias del cemento y de la construcción". Premio "Juan de la Cierva", 1958. 